(2) Open Access Full Text Article

REVIEW

\title{
A stepwise approach for the management of capsular contraction syndrome in hinge-based accommodative intraocular lenses
}

This article was published in the following Dove Press journal:

Clinical Ophthalmology

7 June 2016

Number of times this article has been viewed

\section{Timothy P Page'}

Jeffrey Whitman ${ }^{2}$

'Department of Ophthalmology, Oakland University William Beaumont School of Medicine, Royal Oak, MI, ${ }^{2}$ Key-Whitman Eye Center, Dallas, TX, USA
Correspondence: Timothy P Page Oakland Ophthalmic Surgery, 800 South Adams Road, Suite 20I, Birmingham, MI 48009, USA

Email tpagemd@yahoo.com
Purpose: The aims of this study are to define the various stages of capsular contraction syndrome (CCS) and its effect on refractive error with hinge-based accommodating intraocular lenses (IOLs) and to describe a systematic approach for the management of the different stages of CCS.

Methods: Hinge-based accommodative IOLs function via flexible hinges that vault the optic forward during accommodation. However, it is the flexibility of the IOL that makes it prone to deformation in the event of CCS. The signs of CCS are identified and described as posterior capsular striae, fibrotic bands across the anterior or posterior capsule, and capsule opacification. Various degrees of CCS may affect hinge-based accommodating IOLs in a spectrum from subtle changes in IOL appearance to significant increases in refractive error and loss of uncorrected visual acuity. The signs of CCS and its effect on IOL position and the resulting changes in refractive error are matched to appropriate treatment plans

Results: A surgeon can avoid CCS and manage the condition if familiar with the early signs of CCS. If CCS is identified, yttrium-aluminum-garnet laser capsulotomy should be considered. If moderate CCS occurs, it may be effectively treated with insertion of a capsular tension ring. If CCS is allowed to progress to advanced stages, an IOL exchange may be necessary.

Conclusion: Surgeons should be familiar with the stages of CCS and subsequent interventions. The steps outlined in this article help to guide surgeons in the prevention and management of CCS with hinge-based accommodative IOLs in order to provide improved refractive outcomes for patients.

Keywords: z-syndrome, pseudophakic tilt, IOL subluxation, CTR, capsular tension ring, capsular fibrosis

\section{Introduction}

At the time of cataract surgery, many patients select intraocular lenses (IOLs) to reduce or eliminate their dependency on spectacle correction. To satisfactorily achieve both distance and near uncorrected visual acuity (UCVA), several options are currently available.

Multifocal IOLs may provide excellent distance and near UCVA with spectacle independence in a high percentage of patients. However, multifocal IOLs are known to have some potentially undesirable side effects, preventing many patients from selecting these implants. ${ }^{1,2}$ In addition, a number of patients have preexisting ocular conditions, unrealistic expectations, or low tolerance of risk, which may exclude them from being a good candidate for a multifocal IOL. ${ }^{3}$

Accommodative IOLs are another good option to reduce dependence upon spectacle correction. These IOLs appeal to patients who do not wish to risk side effects 


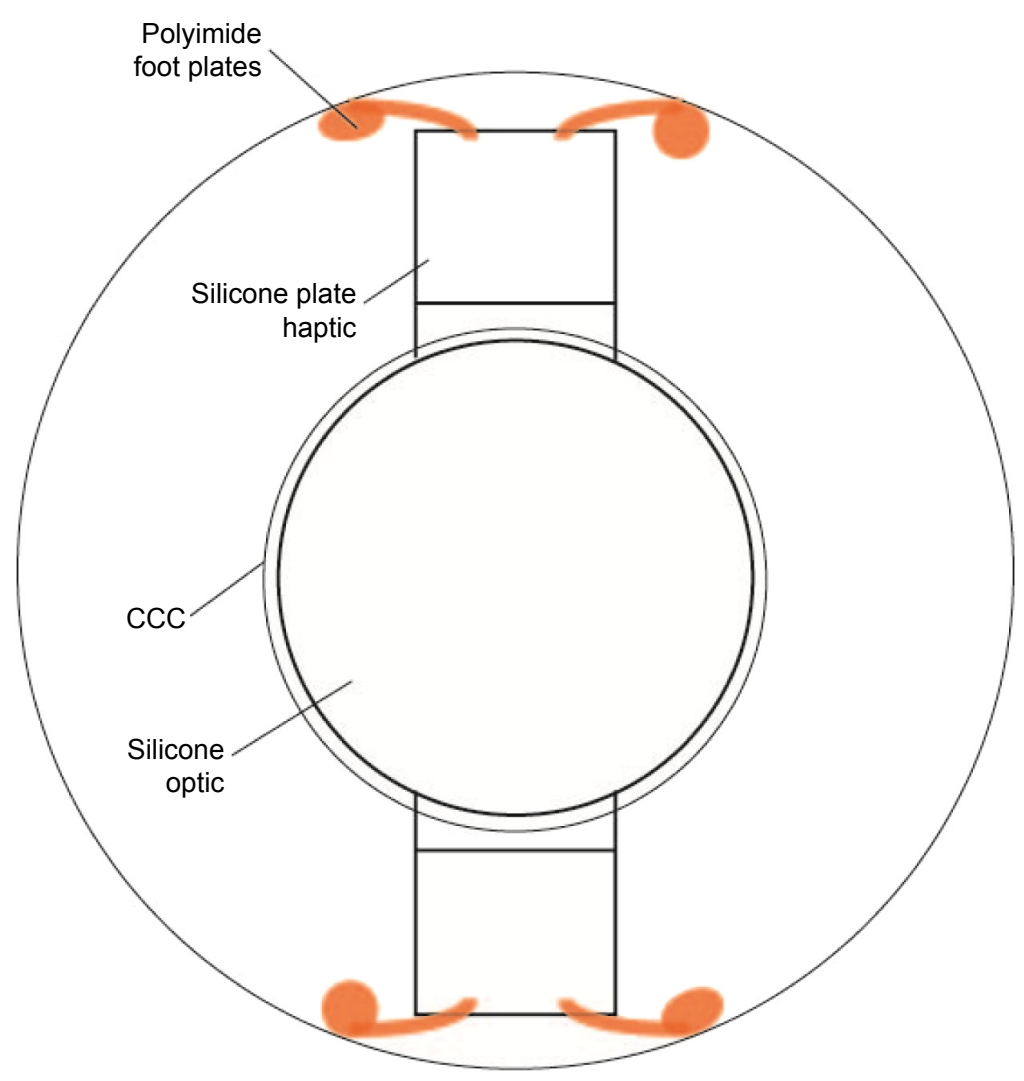

Figure I Ideal position of a hinge-based accommodative IOL in the capsular bag. Abbreviations: CCC, continuous curvilinear capsulorhexis; IOL, intraocular lens.

associated with multifocal IOLs or those who may have some ocular conditions that exclude them from using a multifocal IOL.

The accommodative IOL has the advantage over a multifocal lens in that it does not require diffraction to achieve greater depth of focus. Rather, the accommodative IOL works on the premise of two flexible hinges allowing the central monofocal optic to move forward with accommodation (Figures 1 and 2) The monofocal nature of the optic reduces light scatter, loss of contrast sensitivity, and halos around lights as compared to a multifocal IOL. The monofocality of an accommodating IOL also makes it an attractive option over a multifocal IOL for the growing number of postrefractive surgery patients facing cataract surgery who may have some degree of irregular astigmatism observed with corneal topography or a higher order aberration (HOA) profile excluding them from multifocal IOL use. (This concept is further explained in "Discussion" section.)

While accommodative IOLs may be associated with fewer visual side effects than multifocal IOLs, a number of cases have been reported describing capsular contraction syndrome (CCS) with a negative effect upon visual acuity, with some cases leading to explantation of the IOL. ${ }^{4,5}$

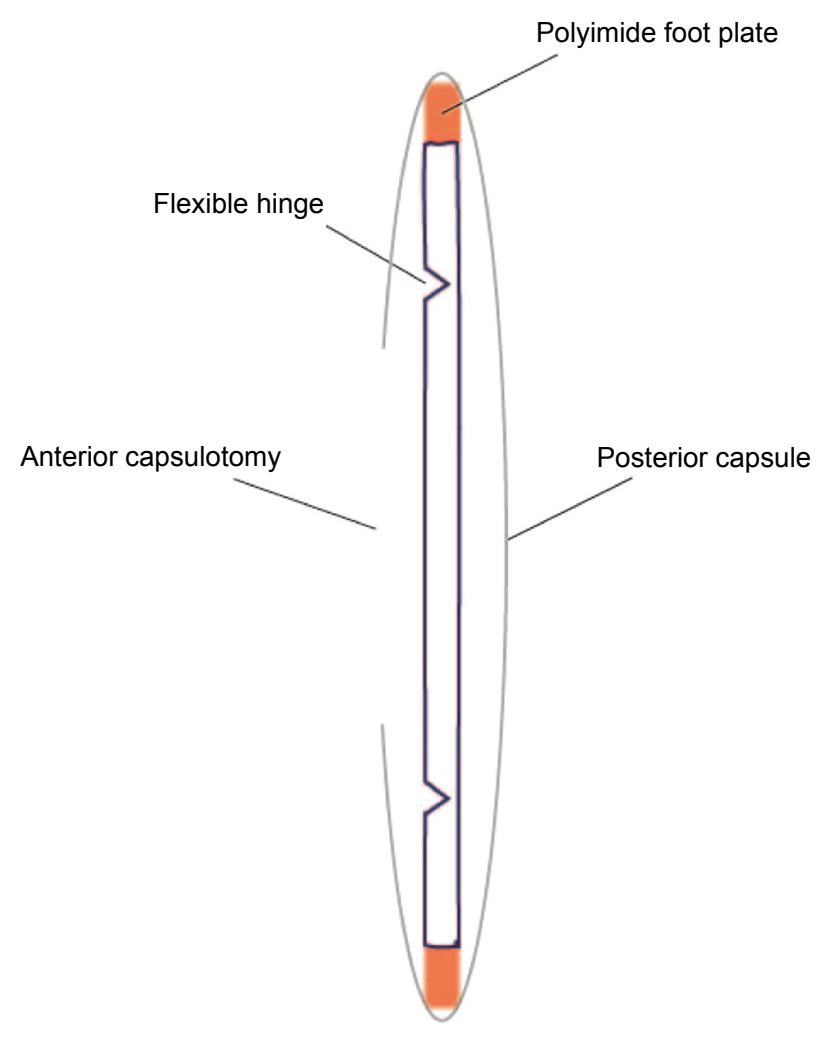

Figure 2 Side profile of a hinge-based accommodative IOL in the capsular bag. Abbreviation: IOL, intraocular lens. 
Table I Examination intervals to avoid capsular contraction syndrome

\begin{tabular}{llllll}
\hline & POD I & POD 7 & POD 30 & POD 60 & POD 90 \\
\hline Examination & SLE & SLE and refraction & $\begin{array}{l}\text { SLE, dilation, and refraction } \\
\text { if UCVA changes }\end{array}$ & $\begin{array}{l}\text { SLE, dilation, and refraction } \\
\text { if UCVA changes }\end{array}$ & $\begin{array}{l}\text { SLE, dilation, and refraction } \\
\text { if UCVA changes }\end{array}$ \\
\hline
\end{tabular}

Abbreviations: POD, postoperative day; SLE, slit lamp examination; UCVA, uncorrected visual acuity.

With appropriate observation and early detection, surgeons can avoid and manage CCS in accommodating IOLs.

In this article, the authors describe a method to avoid CCS and outline a stepwise approach for the management of capsular contraction with accommodative IOLs. The goal of this approach is to allow surgeons to improve surgical outcomes for their accommodative IOL patients, thus avoiding complications and achieving greater spectacle independence for many of them.

\section{Method}

After successful implantation of a hinge-based accommodative IOL, the patient should have standard postoperative slit lamp examinations. The authors typically see patients on postoperative day (POD) \#1, POD \#7, POD \#30, POD \#60, and POD \#90. The IOL and capsular bag should be examined for any signs of fibrosis, striae, or capsular opacification as shown in Table 1. After 90 days, the patient is followed up on an annual basis.

A baseline refraction should be established within the first several weeks to 1 month to monitor the patient for any significant changes in sphere or cylinder. Monthly dilated examinations at postoperative 1 month, 2 months, and 3 months will also allow for the early detection of fibrosis or $\mathrm{CCS}$. If changes in refractive error are occurring during the monthly postoperative visits, the surgeon should consider taking action to prevent a significant fibrotic contraction of the capsule and subsequent malposition of the lens. If fibrotic contraction and malposition with refractive error change occur, we follow a stepwise approach for the management of the condition. The inclusion criteria for initiating an intervention begin with any change in refractive error that appears to be based on fibrosis of the capsule. Mild changes in refractive error due to fibrosis are defined as up to $1.0 \mathrm{D}$ of change in refractive error. Moderate changes in refractive error due to fibrosis are defined as $>1.0$ D. A stepwise approach to refractive error changes induced by capsular contraction is shown in Table 2 .

\section{CCS with $\leq 1.0 \mathrm{D}$ of change}

If the patient's refractive error has a shift of up to $1.0 \mathrm{D}$ in either sphere or astigmatism, the surgeon should be alerted to the possibility of early capsular contraction affecting the
IOL position. If the signs of CCS are present, including fibrotic bands, striae, or capsular opacification (Figures 3 and 4), intervention should be taken as the effect of further contraction can be halted with yttrium-aluminum-garnet (YAG) laser capsulotomy. A YAG capsulotomy may also alleviate some of the contractile forces acting upon the IOL, thus improving the refraction and UCVA. ${ }^{5}$ Three separate posterior capsulotomies are made with the YAG laser as depicted in Figures 5 and 6. A small linear capsulotomy is created distal to the hinge on each haptic, and a central capsulotomy is also created. Care should be taken not to connect these three capsulotomies.

It should be noted that unique presentations may occur with CCS. For example, if a distinct fibrotic band is an obvious source of capsular contraction and the refractive error has changed by $>1.0 \mathrm{D}$, a YAG lysis of the fibrotic band is often sufficient to alleviate the contraction. The potential disadvantage of this maneuver is that it diminishes the possibility of surgical repositioning of the lens and use of a capsular tension ring (CTR) if the YAG fails to correct the problem.

\section{CCS with $>I .0 \mathrm{D}$ of refractive change}

If the patient develops a significant change, defined as $>1.0 \mathrm{D}$ of astigmatism or sphere due to capsular contraction, a YAG capsulotomy may not alleviate the refractive effects of contraction. In this situation, we recommend not performing a YAG laser. Rather, a viscodissection of the fibrosis and rotation of the lens 3 to 4 clock hours (or $180^{\circ}$ for a toric accommodative IOL) with insertion of a CTR will be most effective in correcting the IOL position and preventing further contraction.

Table 2 Stepwise approach for the management of capsular contraction syndrome in a hinge-based accommodative IOL

\begin{tabular}{ll}
\hline $\begin{array}{l}\text { Refractive change determined to be } \\
\text { caused by contraction }\end{array}$ & $\begin{array}{l}\text { Recommended course } \\
\text { of action }\end{array}$ \\
\hline $\begin{array}{l}\text { Induced lenticular astigmatism up to I.O D } \\
\text { Induced lenticular astigmatism >I.O D }\end{array}$ & $\begin{array}{l}\text { YAG capsulotomy } \\
\text { Viscodissection of fibrosis } \\
\text { with CTR insertion }\end{array}$ \\
$\begin{array}{l}\text { Persistent IOL contraction with } \\
\text { IOL exchange } \\
\text { capsulotomy }\end{array}$ & \\
\hline
\end{tabular}

Abbreviations: CTR, capsular tension ring; IOL, intraocular lens; YAG, yttriumaluminum-garnet. 


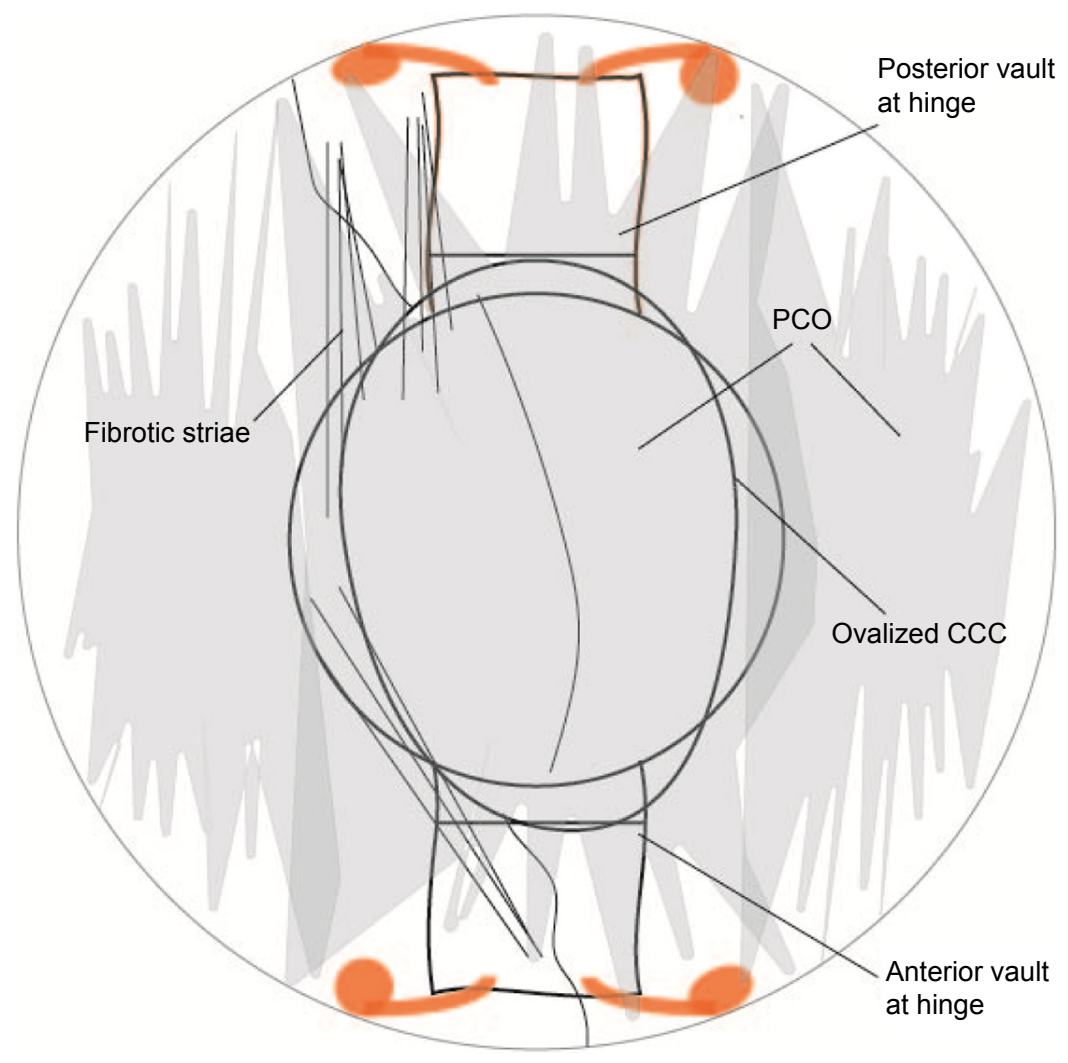

Figure 3 Hinge-based accommodative IOL with PCO, fibrosis, and capsular contraction.

Abbreviations: CCC, continuous curvilinear capsulorhexis; IOL, intraocular lens; PCO, posterior capsular opacity.

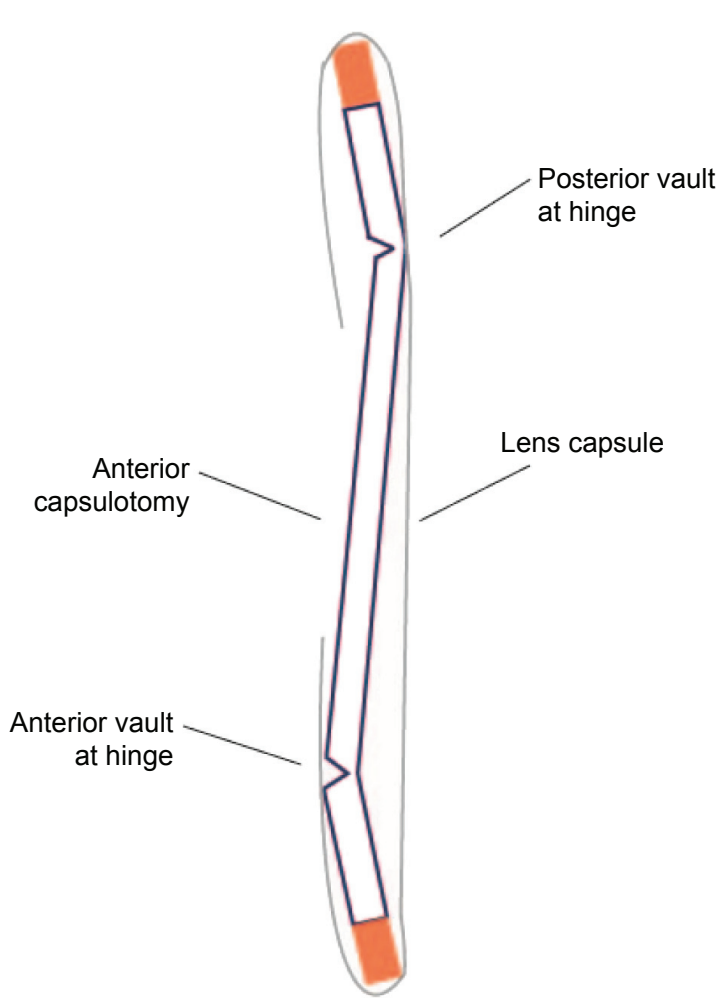

Figure 4 Side profile hinge-based accommodative IOL with fibrosis and capsular contraction.

Abbreviation: IOL, intraocular lens.
The technique for this maneuver is as follows. Under topical or peribulbar anesthesia, a cohesive ocular viscoelastic device (OVD) is injected into the capsular bag to viscodissect any adhesions between anterior and posterior capsular capsules. The IOL will likely be observed to return to its original position during this maneuver. Furthermore, viscodissection should be used to open the entire capsular bag, freeing any fibrosis to the fornix of the bag. When possible, rotation of the IOL several clock hours, or $180^{\circ}$, for a toric accommodating IOL ensures release of adhesions. A standard CTR is then inserted into the capsular bag. Performing these maneuvers prior to 3 months postoperatively can lead to a higher degree of success as fibrosis of the capsule and haptics will be less likely.

If fibrosis or adhesions create difficulty in rotating the IOL, the unique haptic design of the accommodating IOL allows for rotation in either direction. If difficulty is encountered during CTR insertion or displacement of the capsular bag is noted during CTR deployment, the surgeon should retract the CTR and perform further viscodissection. If the leading eyelet of the CTR continues to encounter resistance from either fibrosis or the haptics of the IOL, one may consider retracting the CTR and redirecting it in the other 


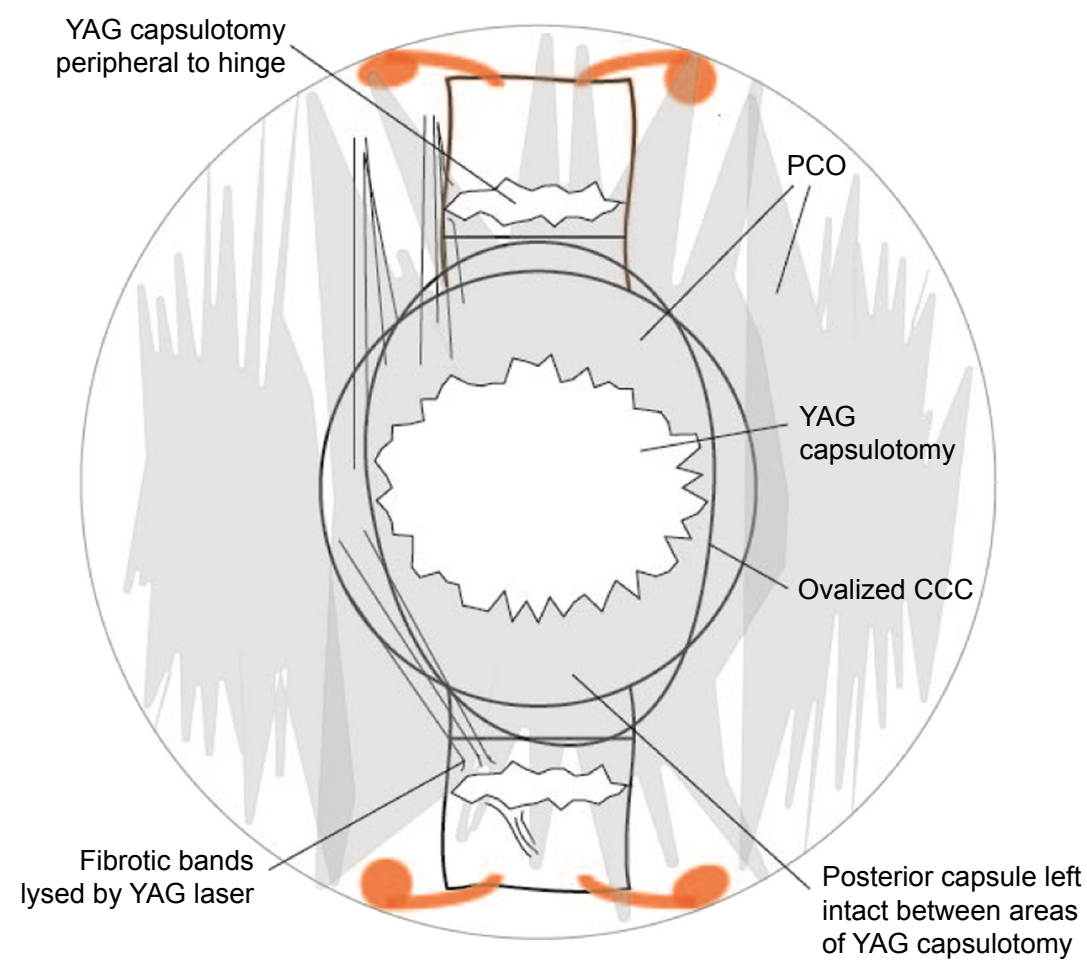

Figure 5 YAG laser capsulotomy for PCO with asymmetric fibrosis and capsular contraction.

Abbreviations: CCC, continuous curvilinear capsulorhexis; PCO, posterior capsular opacity; YAG, yttrium-aluminum-garnet.

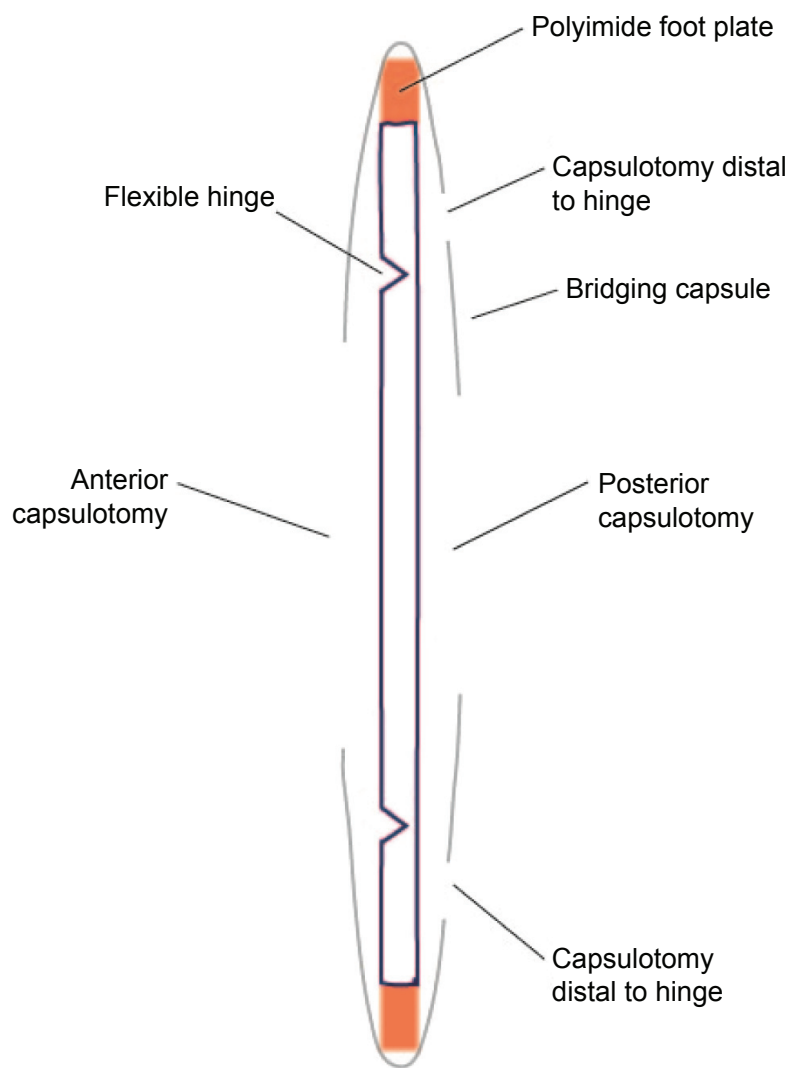

Figure 6 Side profile hinge-based accommodative IOL after YAG laser for fibrosis and capsular contraction.

Abbreviations: IOL, intraocular lens; YAG, yttrium-aluminum-garnet. direction that may bypass the area of resistance. In addition, a simple modification of CTR insertion with a suture-guided technique as previously published may facilitate insertion of the CTR (Video S1). ${ }^{6}$

\section{CCS with YAG failure to achieve desired results}

If the capsule has already undergone YAG capsulotomy without satisfactory resolution of the $\mathrm{Z}$ syndrome, an IOL exchange may be considered. The patient and surgeon should discuss preoperatively whether the patient is a good candidate for a three-piece sulcus placed monofocal versus a three-piece sulcus placed multifocal IOL.

For the IOL exchange technique, a dispersive OVD is injected above and below the IOL to help prevent vitreous prolapse and protect the corneal endothelium. Additional OVD is injected below the IOL and a microscissor suitable for cutting IOLs is used to cut along the plate haptic of the IOL, distal to the hinge if possible. It is important not to pull on the polyimide footplates as they are usually fibrosed into the fornix of the capsular bag and any attempt to explant them may result in an iatrogenic zonular dialysis. After the haptics have been cut, they may be trimmed down with microscissors so that they will not be within the pupillary margin in mesopic or scotopic conditions. The optic may then be grasped with 
lens removal forceps and pulled through a $3.0 \mathrm{~mm}$ incision or can be cut in half and removed.

If vitreous prolapse occurs, the surgeon must perform a vitrectomy following the basic principles of removing any vitreous that is anterior to the posterior capsule, inject dispersive OVD into the sulcus and implant the predetermined three-piece IOL, and remove the OVD and inject a miotic to be sure that the IOL is centered and the pupil is round with no vitreous prolapse (Video S2).

\section{Discussion}

Many patients are electing presbyopia-correcting IOLs at the time of cataract surgery to provide them with less dependence on spectacles. It is incumbent upon ophthalmologists to guide our patients to the best possible IOL choice to meet their visual needs while minimizing potential complications associated with presbyopia-correcting IOLs. Patients should always be advised on the high quality of vision that can be achieved with monofocal IOLs and use of spectacles for near or distance vision. In addition, mono vision with monofocal IOLs should be discussed in the preoperative counseling and may be a satisfactory alternative for many patients. ${ }^{7}$ Almost any IOL option that a patient may choose today has its own set of advantages and disadvantages. A surgeon must exercise great diligence to screen any potential existing ocular conditions that may prevent a patient from realizing the benefits of a multifocal IOL. While multifocal IOLs may provide excellent near and distant UCVA, coexisting macular or corneal conditions may render the patient with unsatisfactory UCVA or even best corrected visual acuity.

In addition, all multifocal IOLs available in the US have negative spherical aberration. It is important to center negative spherical aberration IOLs within the visual axis to prevent higher degrees of coma. ${ }^{8}$ Furthermore, HOAs in the cornea may interfere with the functionality of a multifocal IOL. In our practices, patients are also screened for higher degrees of angle kappa to prevent HOAs associated with malalignment of negative spherical aberration IOLs.

Although the monofocal optic of the accommodating IOL allows a greater number of patients to use the lens, not every patient is a good candidate for these IOLs. Patients with a history of uveitis may be at increased risk for fibrosis and CCS affecting the IOL, and it should be avoided in this subset of patients.

A hinge-based accommodative IOL functions by having flexible hinges vaulting the optic forward during accommodation. However, this flexibility at the hinge is what makes it vulnerable to distortion in the event of capsular contraction.
The surgeon must be vigilant to observe for posterior capsular striae, fibrotic bands, and contraction in the postoperative period. Asymmetric fibrosis in the capsular bag and ovalization of the anterior capsulotomy along the axis of the haptics are often the early signs of contraction.

To avoid CCS, several prevailing concepts should be taken into consideration. First, during cataract surgery, careful attention should be paid to cortical material removal and capsular epithelial cell removal. Careful polishing of the posterior capsule and the underside of the anterior capsular leaflet is important (Video S3). Second, anterior chamber stability with water-tight incision is of paramount importance. A single interrupted 10-0 nylon suture helps to insure a nonleaking stable wound. Finally, prolonged anti-inflammatory agents such as steroids and nonsteroidal anti-inflammatory drugs used for up to 8 weeks help to prevent inflammation and potential ensuing capsular contraction.

If a refractive error change occurs and contraction is suspected but not obvious, diagnostic equipment such as the NIDEK OPD III or iTrace may help to determine IOL tilt or a $\mathrm{Z}$ syndrome.

YAG capsulotomy should be considered at the first signs of fibrosis to prevent further contraction or distortion of the IOL. If the contraction has already created a " $Z$ syndrome" with greater than 1.0 D of induced lenticular astigmatism, the patient should be assessed for any single fibrotic band that may be causing the distortion. If it appears that a single band is causing the contraction, a YAG capsulotomy may be performed, but the surgeon must realize that if the contraction is not alleviated, the possibility of inserting a CTR has been all but eliminated. In addition with an open capsule, it may be necessary to later perform an IOL exchange with an anterior vitrectomy.

With greater degrees of CCS and induced refractive change as defined by $>1.0 \mathrm{D}$, and in the absence of YAG capsulotomy, viscodissecting the capsular bag open and inserting a CTR are highly effective in correcting the IOL position. It is worth mentioning that inserting the CTR earlier in the postoperative period, rather than later, makes it much easier to place the CTR into the capsular bag. A YAG capsulotomy performed 2 weeks or 3 weeks after CTR insertion can aid in preventing any further fibrotic effects on the IOL.

While the incidence of capsular contraction resulting in asymmetric folding of the haptic-optic junction has been reported in the literature as a rare complication, we have managed a number of these cases, ostensibly the largest series of cases published to date, with the techniques described in this article. Our method of managing CCS is based upon the 
Table 3 Lenticular astigmatism $>$ I.0 D managed with reposition and CTR

\begin{tabular}{|c|c|c|c|c|}
\hline Complication & Onset & Intervention & $\begin{array}{l}\text { Preintervention } \\
\text { distance UCVA }\end{array}$ & $\begin{array}{l}\text { Postintervention } \\
\text { distance UCVA }\end{array}$ \\
\hline Z-position & POD 30 & Reposition with CTR & $20 / 50$ & $20 / 20$ \\
\hline Anterior vault & POD 14 & Reposition with CTR & $20 / 30$ & $20 / 20$ \\
\hline Z-position & POD 30 & Reposition with CTR & $20 / 60-2$ & $20 / 25$ \\
\hline Superior haptic vault & POD 90 & Reposition with CTR & $20 / 70$ & $20 / 20$ \\
\hline Superior optic tilt & POD 42 & Reposition with CTR & $20 / 60$ & $20 / 25$ \\
\hline Slight anterior vault & POD 30 & Reposition with CTR & $20 / 50$ & $20 / 25$ \\
\hline Temporal haptic vault & POD 30 & Reposition with CTR & $20 / 150$ & $20 / 30$ \\
\hline Nasal haptic vault & POD 42 & Reposition with CTR & $20 / 400$ & $20 / 25$ \\
\hline Anterior vault & POD 30 & Reposition with CTR & $20 / 400$ & $20 / 100^{\mathrm{a}}$ \\
\hline Posterior vault & POD 42 & Reposition with CTR & $20 / 70-2$ & $20 / 200^{\mathrm{b}}$ \\
\hline Temporal haptic vault & POD 42 & Reposition with CTR & $20 / 40$ & $20 / 70^{c}$ \\
\hline Temporal haptic vault & POD 120 & Reposition with CTR & $20 / 60$ & $20 / 40$ \\
\hline Temporal haptic vault & POD 49 & Reposition with CTR & $20 / 60$ & $20 / 25$ \\
\hline
\end{tabular}

Notes: aContraction and vault reoccurred, CTR removed with lens reposition with resulting myopic refractive error. ${ }^{\mathrm{b}}$ Eye became myopic and was further treated with a piggyback IOL for 20/20 UCVA. 'Eye became myopic after reposition, patient elected to remain myopic for near vision.

Abbreviations: CTR, capsular tension ring; IOL, intraocular lens; POD, postoperative day; UCVA, uncorrected visual acuity.

degree of refractive error change from baseline to determine intervention with YAG laser or CTR. This may present a challenge as accurate measurement of pseudophakic lenticular astigmatism or refractive change may be difficult to determine in cases where contraction of the capsule results in irregular astigmatism, capsular fibrosis, opacification, and displacement of the optic. A surgeon might not be able to rely solely upon the refractive error change to determine the appropriate intervention and should evaluate the degree of vaulting of the haptic optic junction. For example, if UCVA has significantly decreased and is consistent with displacement of the optic or a large degree of vaulting, a YAG capsulotomy is unlikely to correct the contracted IOL and a CTR would be the intervention of choice. Table 3 illustrates eyes with CCS and refractive error changes of $>1.0 \mathrm{D}$ with subsequent change in UCVA that have been managed with this technique with IOL repositioning and CTR implantation.

In the event where a YAG capsulotomy has already been done and failed to alleviate the CCS, an IOL exchange should be considered. If an IOL exchange is performed, it is important to remember that the polyimide haptics may be left in the capsular fornix and not pulled from the fibrotic fornix to avoid an iatrogenic zonular dialysis. If there are no contraindications to use a multifocal IOL, a three-piece multifocal may be placed in the sulcus to give the patient the increased range of vision they may have had with the accommodative IOL. Although the goal is to prevent an IOL exchange by using the steps outlined in this article, we have had two patients present with YAG capsulotomies that failed to correct the CCS and required an IOL exchange (Table 4).

\section{Limitations}

The limitation of this study is that it is presented as a technique and a case series utilizing a methodical approach for the management of an IOL complication. Further study comparing our technique to an alternative management in a randomized comparative prospective cohort study would be beneficial to determine best practices in the management of CCS. However, with the relatively low incidence of CCS, such a study may be difficult to collect enough data for statistical significance. Furthermore, comparative studies to determine the prophylactic placement of a CTR at the time of cataract surgery and accommodative IOL implantation would be of great interest. Finally, the role of inflammation and the use of anti-inflammatory medications in terms of both potency and duration of use in the postoperative period would be an area of useful research to prevent CCS.

Table 4 Lenticular astigmatism $>2.0 \mathrm{D}$ status post-YAG capsulotomy

\begin{tabular}{lllll}
\hline Complication & Onset & Intervention & $\begin{array}{l}\text { Preintervention } \\
\text { distance UCVA }\end{array}$ & $\begin{array}{l}\text { Postintervention } \\
\text { distance UCVA }\end{array}$ \\
\hline Inferior haptic vault & POD 63 & IOL exchange multifocal & $20 / 200$ & $20 / 25$ \\
Posterior vault & POD 35 & IOL exchange multifocal & $20 / 70$ & $20 / 70$ \\
\hline
\end{tabular}

Abbreviations: IOL, intraocular lens; POD, postoperative day; UCVA, uncorrected visual acuity; YAG, yttrium-aluminum-garnet. 


\section{Conclusion}

In our experience, following this methodical approach for the avoidance and treatment of capsular contraction has led to excellent surgical and patient satisfaction outcomes with accommodative IOLs. As the ability to provide superior refractive results with increasing spectacle independence continues to improve, we must remain vigilant in our IOL selections for each individual patient and be prepared to manage any potential complications. For patients choosing accommodative IOLs, the unlikely event of CCS is best avoided with careful cortical and epithelial cell removal with postoperative anti-inflammatory agents and early detection and treatment of the contraction.

\section{Acknowledgments}

This is to certify that this article has not been previously presented in a meeting. The authors did not receive any financial support from any public or private sources.

\section{Disclosure}

The authors are consultants for products mentioned in this article. TP Page is a consultant for Abbott Medical Optics Inc., Alcon, and Bausch and Lomb Surgical. J Whitman is a consultant for Bausch and Lomb, Alcon, Oasis, Revision
Optics, and Staar Surgical. The authors report no other conflicts of interest in this work.

\section{References}

1. Javitt JC, Steinert RF. Cataract extraction with multifocal intraocular lens implantation: a multinational clinical trial evaluating clinical, functional, and quality-of-life outcomes. Ophthalmology. 2000;107(11): 2040-2048.

2. Gierek-Ciaciura S, Cwalina L, Bednarski L, Mrukwa-Kominek E. A comparative clinical study of the visual results between three types of multifocal lenses. Graefes Arch Clin Exp Ophthalmol. 2010;248(1):133-140.

3. Braga-Mele R, Chang D, Dewey S, et al; ASCRS Cataract Clinical Committee. Multifocal intraocular lenses: relative indications and contraindications for implantation. J Cataract Refract Surg. 2014;40(2): 313-322.

4. FDA [webpage on the Internet]. Manufacturer and User Facility Device Experience (MAUDE) Database. Available from: http://www.accessdata. fda.gov/scripts/cdrh/cfdocs/cfMAUDE/Search.cfm. Accessed April 6, 2016.

5. Yuen L, Trattler W, Boxer Wachler BS. Two cases of Z syndrome with the Crystalens after uneventful cataract surgery. $J$ Cataract Refract Surg. 2008;34(11):1986-1989.

6. Page TP. Suture-guided capsular tension ring insertion to reduce risk for iatrogenic zonular damage. J Cataract Refract Surg. 2015;41(8): $1564-1567$.

7. Zhang F, Sugar A, Jacobsen G, Collins M. Visual function and patient satisfaction: comparison between bilateral diffractive multifocal intraocular lenses and monovision pseudophakia. J Cataract Refract Surg. 2011;37(3):446-453.

8. Holladay JT, Piers PA, Koranyi G, van der Mooren M, Norrby NE. A new intraocular lens design to reduce spherical aberration of pseudophakic eyes. J Refract Surg. 2002;18(6):683-691.
Clinical Ophthalmology

\section{Publish your work in this journal}

Clinical Ophthalmology is an international, peer-reviewed journal covering all subspecialties within ophthalmology. Key topics include: Optometry; Visual science; Pharmacology and drug therapy in eye diseases; Basic Sciences; Primary and Secondary eye care; Patient Safety and Quality of Care Improvements. This journal is indexed on

Submit your manuscript here: http://www.dovepress.com/clinical-ophthalmology-journal

\section{Dovepress}

PubMed Central and CAS, and is the official journal of The Society of Clinical Ophthalmology (SCO). The manuscript management system is completely online and includes a very quick and fair peer-review system, which is all easy to use. Visit http://www.dovepress.com/ testimonials.php to read real quotes from published authors. 